\title{
Encapsulation of food active ingredients in liposomes
}

\begin{abstract}
Liposomes are microscopic vesicles composed of phospholipid bilayers entrapping one or more aqueous compartments. Their properties have permitted numerous applications in several fields, including the food industry. The controlled release of active ingredients can be achieved using liposomes. In this mini-review we briefly introduce various aspects of liposomes preparation and their application in food technology.
\end{abstract}

Volume 8 Issue 3 - 2018

\author{
Padilla Camberos Eduardo,Villanueva \\ Fernando Jesus Emmanuel \\ Medical and Pharmaceutical Biotechnology, Center for Research \\ and Applied Technology in Jalisco, Mexico
}

\begin{abstract}
Correspondence: Padilla Camberos Eduardo, Medical and Pharmaceutical Biotechnology, Center for Research and Applied Technology in Jalisco, ciatej, Normalistas 800 , Guadalajara, Jalisco, Mexico,Tel 3333455200,Email epadilla@ciatej.mx
\end{abstract}

Received: April 18, 2018 | Published: May II, 2018

\section{Introduction}

Liposomes are colloidal aggregates formed by one or more closed bilayers, which include in their interior a small fraction of the aqueous medium. ${ }^{1}$ They form spontaneously when the lipid molecules (mostly phospholipids) generate the membrane that encloses an active agent, and are dispersed in the aqueous medium giving rise to suspensions of vesicles with diameters that can have a size from nanometers to microns (Figure 1). ${ }^{2}$ Liposomes have particular characteristics and have been studied in many fields as medical, environmental and food, some studies are based in models of cell membranes helping to elucidate the physical and chemical behavior, organization of cell compartments and the structures of cell transport. ${ }^{3}$

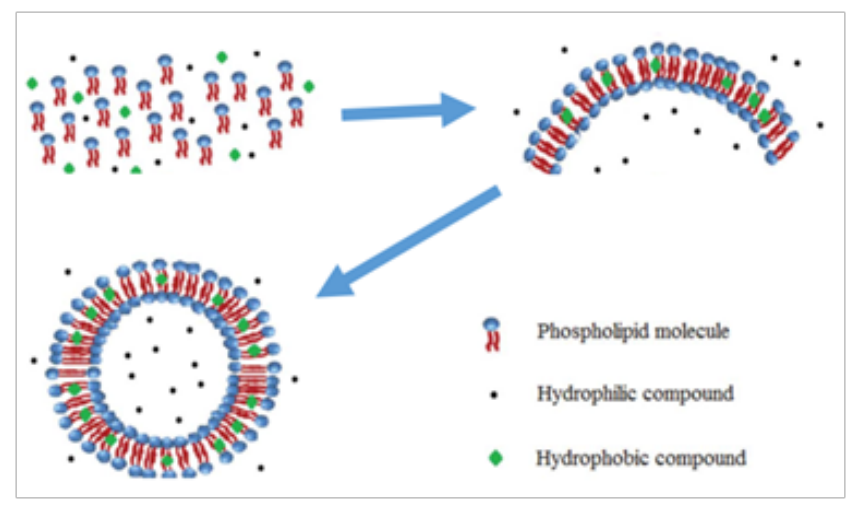

Figure I Liposome formation. modified from Emami et al. ${ }^{12}$

\section{Liposome classification}

Liposomes are classified according their structure, taking account the type of layers formed at the moment of producing the membranes of the vesicles. They surround the active medium that is intended to be encapsulated and the size they have at the end of the encapsulation. ${ }^{4}$

a) Large multilamellar vesicles (LMV): Formed by multiple concentric bilayers separated by an aqueous space of approximately $5 \mathrm{~nm}$ in thickness.

b) Large unilamellar vesicles (LUV): Presents a single lipid bubble and the size ranging from 50 to $300 \mathrm{~nm}$. c) Small unilamellar vesicles (SUV): Measure oscillates from 25 to $50 \mathrm{~nm}$ of diameter. Due to their size they have a very narrow radius of curvature that makes the lipids tend somewhere more in the outer half than in the inner half of the room.

\section{Obtaining liposomes}

There are different methods for obtaining liposomes, by example

i. Method of hydration of the film: when phospholipids and membrane components are dissolved in a volatile organic solvent, evaporation is carried out at reduced pressure to achieve the formation of a thin film on the walls of the evaporation vessel. The rehydration of the film with an aqueous solution leads to the formation of liposomes.

ii. Rapid injection method in ethanol or ether: Phospholipids are dissolved in ethanol or ether with injection to high speed using a syringe over a large volume of aqueous phase. SUV type liposomes form spontaneously.

iii. Detergent removal method: by removing a detergent from a previously prepared suspension of mixed detergent/phospholipid micelles, the formation of SUV liposomes occurs.

iv. Reverse phase evaporation method: a small volume of the aqueous phase is added to an organic solution with the phospholipids and then a sonication treatment to form an emulsion. When the organic phase is eliminated under reduced pressure, the formation of liposomes occurs. ${ }^{5}$

\section{Application of liposomes for food active ingredients}

The lipid double layer structure presented by liposomes are suitable for a correct permeability towards plasma membranes of certain eukaryotic cells and microorganisms. ${ }^{6}$ Liposomes in addition, provide a way to deliver hydrophobic agents, and can also improve the properties of the encapsulated active agent by increasing circulation time. $^{\text {? }}$

The liposomal encapsulation allows protection to the content from enzymatic degradation, which is very common in natural compounds that undergo a rapid degradation as it is the case of the essential oils. ${ }^{7}$ Some articles report that the encapsulation of essential oils trough 
liposomes has allowed a chemical stability of them, as well as an efficient activity as antimicrobial agent for food preservation. ${ }^{8}$

Liposomes have the ability to increase the antimicrobial activities of encapsulated ingredients. For example, liposomes loaded with pure carvacrol and thymol were effective as preservative and conserving agents in food industries.

Another use of liposomes is encapsulation of vitamins to protect and increase the absorption, enhance the bioavailability; for example vitamin $\mathrm{C}$ encapsulated in liposomes retains $50 \%$ of its activity even after 50 days of storage in refrigeration, whereas non-encapsulated vitamin loses its activity after 19 days.

Liposomes have been used in the food industry to improve the flavor of ripened cheese by synergistic delivery of ascorbic acid and tocopherols. Another example of the application is the encapsulation of calcium lactate in lecithin liposomes to fortify the soymilk with levels of calcium equivalent to those found in cow's milk. ${ }^{10}$

Liposomal encapsulation was used to retain the antioxidant activity of resveratrol and recent studies reported a faster rate and better absorption of encapsulated curcumin, a natural polyphenolic phytochemical. ${ }^{11}$

\section{Conclusion}

i. There are many potential applications for liposomes in the food industry, ranging from the protection of sensitive ingredients to increase the efficacy of food additives.

ii. Due to the fast degradation of essential oils, they tend to lose their antimicrobial and antioxidant properties, that's why liposomal encapsulation is a perfect alternative to avoid these problems and delivery them in a better way.

iii. Liposome encapsulation provides multiple benefits to essential oil properties, when they are used as antimicrobials to have an effective control of certain pathogenic microorganisms in foods.

\section{Acknowledgments}

None.

\section{Conflict of interest}

Author declares there is no conflict of interest.

\section{References}

1. Bozzuto G, Molinari A. Liposomes as nano medical devices. Int $J$ Nanomedicine. 2015;10:975-999.

2. Berekaa MM. Nanotechnology in food industry; advances in food processing, packaging and food Safety. Int J Curr Microbiol App Sci. 2015;4(5):345-357.

3. Laouini A, Jaafar-Maalej C, Limayem-Blouza I, et al. Preparation, characterization and applications of liposomes: state of the art. Journal of colloid Science and Biotechnology. 2012;1(2):147-168.

4. Valenzuela C, Hernández V, Rodríguez F, et al. Tecnología de encapsulación y su aplicación en ciencias veterinarias. Avances en Ciencias Veterinarias. 2013;28(2):58.

5. Lanio ME, Luzardo MC, Laborde R, et al. Liposomal vesicles: obtaining, properties and potential applications in biomedicine. Rev Cub Física. 2009;26(1):23-30.

6. Sherry M, Charcosset C, Fessi H, et al. Essential oils encapsulated in liposomes: a review. J Liposome Res. 2013;23(4):268-275.

7. Sebaaly C, Jraij A, Fessi H, et al. Preparation and characterization of clove essential oil-loaded liposomes. Food chemistry. 2015;178:52-62.

8. Sinico C, De Logu A, Lai F, et al. Liposomal incorporation of Artemisia arborescens L. essential oil and in vitro antiviral activity. Eur J Pharm Biopharm. 2005;59(1):161-168.

9. Liolios CC, Gortzi O, Lalas S, et al. Liposomal incorporation of carvacro and thymol isolated from the essential oil of Origanum dictamnus L. and in vitro antimicrobial activity. Food chemistry. 2009;112(1):77-83.

10. Marsanasco M, Chiaramoni NS, del Valle Alonso S. Liposomes as Matrices to Hold Bioactive Compounds for Drinkable Foods: Their Ability to Improve Health and Future Prospects. In Functional FoodImprove Health through Adequate Food. In Tech. 2017.

11. Emami S, Azadmard-Damirchi S, Peighambardoust SH, et al. Liposomes as carrier vehicles for functional compounds in food sector. Journal of Experimental Nanoscience. 2016;11(9):737-759. 